\title{
Registro em prontuário: reflexões sobre a qualidade documental na terapia ocupacional
}

\section{Medical record: reflections on documental quality in occupational therapy}

\author{
Tatiana Barbieri Bombarda ${ }^{1}$, Regina Helena Vitale Torkomian Joaquim²
}

http://dx.doi.org/10.11606/issn.2238-6149.v30i2p110-115

Bombarda TB, Joaquim RHVT. Registro em prontuário: reflexões
sobre a qualidade documental na terapia ocupacional. Rev Ter Ocup Univ São Paulo. 2020 maio-ago.;30(2):110-15.

\begin{abstract}
RESUMO: Os registros de Terapia Ocupacional têm por finalidade descrever o processo terapêutico tornando explícito o pensamento crítico, o raciocínio profissional e a tomada de decisões do terapeuta ocupacional. Contudo, a importância do prontuário vai além do âmbito assistencial, visto essas anotações também ser utilizadas no contexto administrativo, educacional e científico. Mas o desempenho deste procedimento é realizado com qualidade pelos terapeutas ocupacionais? Visando discorrer sobre o tema, serão apresentados pelos autores apontamentos sobre a qualidade dos registros, a partir de uma análise reflexiva norteada pelas diretrizes da profissão sobre documentação. Como principais apontamentos, pode-se afirmar que a prática documental dos terapeutas ocupacionais ocorre de modo inconsistente, fator vinculado ao desconhecimento das diretrizes da profissão, à dificuldades com a linguagem técnica e ao processo de formação inicial e continuada. Recomenda-se a ampliação e aprofundamento de pesquisas sobre o tema para obtenção de estratégias educacionais mais eficazes e sistematizações de modelos de registros melhor gerenciáveis.
\end{abstract}

DESCRITORES: Registros; Controle de formulários e registros/ normas; Terapia ocupacional; Documentação.
Bombarda TB, Joaquim RHVT. Medical record: reflections on documental quality in occupational therapy. Rev Ter Ocup Univ São Paulo. 2020 May-Aug.;30(2):110-15.

\begin{abstract}
The records of occupational therapy aim to describe the therapeutic process, making explicit the critical thinking, professional reasoning and decision making of the occupational therapist. However, the importance of record goes beyond the scope of care, since these notes are also used in the administrative, educational and scientific context. But is the performance of this procedure performed with quality by occupational therapists? Aiming to discuss the theme, the authors will present informations on the quality of the records, based on a reflexive analysis guided by the profession's guidelines on documentation. As main datas, the presence of inconsistencies in the documentary practice of occupational therapists were evidenced, factor is related to the lack of knowledge of the guidelines of the profession, the difficulties with the technical language and the process of initial and continued training. Therefore, it is recommended to expand and deepen researches on the tematic, to obtain more effective educational strategies and systematization of more manageable records models.
\end{abstract}

KEYWORDS: Records; Forms and records control/standards; Occupational therapy; Documentation.

Artigo da tese de doutorado "Registro em prontuário: compreensão do processo de ensino aprendizagem no âmbito da Terapia Ocupacional em contextos hospitalares”, de Tatiana Barbieri Bombarda. Orientação Regina H.V. Torkomian Joaquim, Programa de Pós-Graduação em Terapia Ocupacional da Universidade Federal de São Carlos.

1. Professora Adjunta do Departamento de Terapia Ocupacional da Universidade Federal de São Carlos. ORCID: https://orcid.org/ 0000-0002-9478-7945. E-mail: tatibb_to@yahoo.com.br.

2. Professora Associada do Departamento de Terapia Ocupacional da UFSCar. Docente credenciada no Programa de Pós-Graduação em Terapia Ocupacional da Universidade Federal de São Carlos. ORCID: https:/orcid.org/0000-0003-3700-397X. E-mail: joaquimrhvt@gmail.com. Endereço para correspondência: Departamento de Terapia Ocupacional - DTO/UFSCar. Universidade Federal de São Carlos - UFSCar. Rodovia Washington Luis, km 235 - São Carlos, SP, BR. CEP: 13565-905. E-mail: tatibb_to@yahoo.com.br. 


\section{INTRODUÇÃO}

$\mathrm{O}$ prontuário configura-se como um documento importante na área da saúde por dispor de informações necessárias para assegurar a continuidade e qualidade do tratamento prestado ${ }^{1}$.

No âmbito da Terapia Ocupacional, o registro em prontuário constitui-se como um procedimento obrigatório ${ }^{2,3}$ em que se preconiza anotações sistematizadas de forma organizada, objetiva e precisa, com informações concisas e breves ${ }^{4}$. Têm por finalidade descrever o processo terapêutico tornando explícito o pensamento crítico, o raciocínio profissional e a tomada de decisões do terapeuta ocupacional. As informações devem estar atualizadas e legíveis e o paciente tem o direito em acessar as informações a quaisquer tempo ${ }^{5}$.

O prontuário permite a sistematização das informações de modo cronológico sobre o estado do paciente e possibilita parametrização dos resultados obtidos a partir da assistência ofertada ${ }^{3}$. Registros de qualidade facilitam a comunicação com pacientes, cuidadores, familiares e outros profissionais de saúde e podem ser utilizados para o desenvolvimento de pesquisas e como base da educação profissional ${ }^{5}$. Sendo assim, o prontuário apresenta-se como ferramenta importante para o contexto administrativo, educacional e científico, além da assistência ${ }^{6,7}$.

Diante do exposto, considerando a relevância do registro em prontuário, este artigo visa apresentar informações sobre a qualidade documental na Terapia Ocupacional e discorrer sobre os tensionamentos desta prática a partir de dados provenientes da literatura científica.

Para a descrição do ponto de vista dos autores, optou-se pela apresentação de algumas pesquisas que se debruçaram sobre o tema como estratégia de fundamentar os apontamentos reflexivos aqui elaborados. Como arcabouço teórico foram utilizadas as diretrizes da profissão sobre registros e documentação. Os documentos utilizados consistiram no Guidelines for Documentation of Occupational Therapy, elaborado pela American Occupational Therapy AssociationAOTA $^{3}$, na Resolução 415 do Conselho Federal de Fisioterapia e Terapia Ocupacional ${ }^{2}$, no Practice Guideline: client records, elaborado pelo College of Occupational Therapists of Ontario ${ }^{8}$ e Practice Standards for Managing Client Information do College of Occupational Therapists of British Columbia ${ }^{5}$.

\section{Qualidade documental: apontamentos de dados empíricos}

Estudos sobre a temática dos registros em Terapia Ocupacional são escassos, quando comparados à produção nas áreas de Medicina e Enfermagem, por exemplo, e indicam inconsistências na realização deste procedimento, denotando a necessidade de olhares mais criteriosos dos profissionais.

Os estudos produzidos em diferentes países incluem apresentações de modelos de registros formulados para um público e/ou serviço específico, apresentação de dados que relacionam a documentação com níveis de insatisfação profissional, avaliação da qualidade documental e da relação existente entre anotações em prontuário e produção de evidências.

Pesquisa realizada na Suécia avaliou os significados atribuídos à documentação adequada pela ótica de 11 terapeutas ocupacionais. Foram realizadas entrevistas com os participantes, sendo evidenciadas nos resultados cinco contradições no processo de produção de registro: 1) contradições entre linguagem cotidiana e linguagem profissional; 2) entre documentação altamente estruturada e aquela que comunica uma visão global do paciente; 3) entre as exigências jurídicas e éticas e as condições do ambiente de trabalho; 4) entre as diferentes expectativas dos diversos destinatários dos registros e 5) entre raciocínio clínico de terapia ocupacional e raciocínio clínico médico. Diante de tais dados, mesmo considerando que a amostra reduzida seja um fator limitante do estudo, as autoras enfatizaram sobre necessidade de investimentos na linguagem técnica profissional ${ }^{9}$.

Em complemento, o estudo de Pierre ${ }^{10}$ avaliou 22 prontuários de Terapia Ocupacional e realizou entrevistas com 11 profissionais, com o objetivo de verificar práticas valorizadas pelos terapeutas ocupacionais em sua rotina de trabalho, mas não registradas em prontuário. $\mathrm{O}$ estudo utilizou como referencial teórico o código de ética da profissão e documentos jurídicos suecos. Os resultados obtidos denotaram como práticas valorizadas, mas não registradas, informações sobre o foco no desempenho ocupacional, supervisão das atividades de vida diária de modo informal, circunstâncias não entendidas como atendimento propriamente dito, aquisição de dados úteis ao processo terapêutico e oferta de apoio emocional. A razão manifestada para a não documentação destes domínios atrelase a dificuldades de nomeações pelos terapeutas ocupacionais no emprego da linguagem técnica, fator configurado pelos autores como desafio para a profissão que demanda estabelecimento de uma "linguagem da ocupação"10.

Já no estudo desenvolvido por Hedberg-Kristensson e Iarwsson ${ }^{11}$, foi realizada comparação da qualidade documental produzida por terapeutas ocupacionais em dois equipamentos de saúde localizados em distintos municípios da Suécia. Os serviços apresentavam rotinas de registros diferentes, visto o uso em um deles do prontuário físico e, em outro, do prontuário eletrônico. Em específico, buscou-se avaliar a qualidade das informações atreladas às prescrições de 
tecnologia assistiva. Foram analisados 182 registros, sendo constatados déficits na qualidade da documentação em ambos os serviços. Foi verificada ausência de anotações referentes a diferentes itens avaliados e presença de informações inconsistentes, assim como fragilidades nos dados referentes à prescrição de tecnologia assistiva. Frente aos achados os autores discorreram sobre a importância documental no processo de reabilitação e sinalizaram como demanda a necessidade de investimentos no processo de formação dos terapeutas ocupacionais para a prática da documentação com melhores índices de qualidade ${ }^{11}$.

No contexto sul-africano Rischmuller e Fanzsen ${ }^{12}$, apresentaram na primeira etapa do estudo o desenvolvimento de checklist para auditar registros clínicos de terapia ocupacional no campo da educação e para avaliação da qualidade de registros produzidos por terapeutas ocupacionais atuantes em escolas. Posteriormente os autores avaliaram 76 registros produzidos por terapeutas ocupacionais atuantes em quatro escolas, que foram selecionados por amostragem aleatória. A análise dos dados foi feita por estatística descritiva, sendo indicado nos resultados que nenhuma das seções analisadas obteve $100 \%$ dos itens preconizados (menos de 50\% das informações das seções foram incluídas nos registros). Os autores expressaram que registros incompletos influenciam na qualidade da intervenção prestada pelo terapeuta ocupacional, sendo destacado a dificuldade do profissional em fornecer informações sobre progressos obtidos, pontos fortes e frágeis de desempenho do aluno, informações importantes e requeridas para a continuidade assistencial no ano escolar seguinte ${ }^{12}$.

No Brasil, o estudo "O registro de práticas interventivas da Terapia Ocupacional na educação inclusiva" visou entre seus objetivos à identificação de dados sobre a prática dos registros em prontuário no âmbito escolar. Os participantes foram 55 terapeutas ocupacionais atuantes no processo de educação inclusiva e cadastrados no Conselho Regional de Fisioterapia e Terapia Ocupacional da $3^{\text {a }}$ Região-CREFITO-3. Os resultados demonstraram o uso da narrativa livre como o modelo de registro mais utilizado na documentação e presença de fragmentações das informações relacionadas ao processo de guarda das anotações. Tais dados evidenciaram fragilidade na sistematização dos registros, sendo apontado pelas autoras a necessidade do início de produções científicas brasileiras centradas nesta temática ${ }^{13}$.

Apresentando dados provenientes de análise documental, o artigo "O registro da intervenção no prontuário pelo terapeuta ocupacional em um ambulatório infanto-juvenil", apontou dados sobre o conteúdo das informações registradas em prontuário, efetivados por terapeutas ocupacionais em um ambulatório localizado no interior do estado de São Paulo. Para a coleta de dados foi utilizado um checklist, sendo realizada análise documental de 15 prontuários, abrangendo 269 registros de evoluções. Ao final do estudo, foram apontadas fragilidades na documentação analisada como a ausência de informações preconizadas pelas diretrizes nacionais ${ }^{14}$.

Concernente a comunicação de evidências na documentação Davis et al. ${ }^{15}$ desenvolveram um estudo com a proposta de investigar o uso da Prática Baseada em Evidência (PBE) e a comunicação da evidência na documentação, sob a ótica de 126 terapeutas ocupacionais da região centro-oeste dos Estados Unidos.

Os resultados demonstraram que aspectos como limitação de tempo, requisitos de cotas de pacientes e falta de compreensão de pesquisas publicadas constituem-se como barreiras ao uso da PBE, bem como interferem na citação da evidência na documentação. A comunicação da evidência na documentação foi considerada importante quando relacionada a situações de financiamento pelo serviço prestado e/ou quando determinado pelos departamentos/instituições, todavia, a principal dificuldade elencada no processo de comunicação de evidências nos registros consistiu na ausência de modelos ou diretrizes para sua execução ${ }^{15}$.

Mais recentemente, o estudo "Practice-based evidence: evaluating the quality of occupational therapy patient records as evidence for practice" apresentou a análise de 240 registros realizados por 49 terapeutas ocupacionais atuantes em instituições de saúde de uma região da África do Sul. A auditoria nos registros foi realizada por meio do uso de checklist formulado pelos pesquisadores e fundamentado a partir de revisão sistemática ${ }^{16}$.

Entre os resultados os autores verificaram inconsistências nas informações sobre avaliação da linha de base e informações referentes à reavaliação, fato apontado como entrave para a determinação da eficácia da assistência em terapia ocupacional. A baixa pontuação obtida na auditoria demonstrou que os registros não contêm informações suficientes para produzir dados robustos para a criação de evidências científicas, sendo sinalizado pelos autores a necessidade de ser concebidas maneiras gerenciáveis de documentar a prática da terapia ocupacional ${ }^{16}$.

\section{Tensionamentos e desafios atuais}

Na década de 1980, Gillete ${ }^{17}$ abordou a importância da documentação e a relação direta entre a fragilidade das informações registradas com a desvalorização do trabalho terapêutico ocupacional, com implicações para o pagamento dos serviços prestados e para contribuição do desenvolvimento de pesquisas que produzam evidências científicas sobre a prática da Terapia Ocupacional. 
Todavia, estudos ${ }^{12,13,14,16}$ atuais mostram que fragilidades nos registros em prontuário ainda se configuram como problema no âmbito da Terapia Ocupacional, visto indicações de práticas que envolvem informações inconsistentes no procedimento da documentação em prontuário.

Entende-se que o problema das inconformidades dos registros está vinculado em especial a três aspectos: ao desconhecimento das diretrizes da área, às dificuldades com a linguagem técnica e ao processo de formação.

Em diferentes países, as diretrizes profissionais sobre a temática, embora baseadas em referenciais teóricos diferentes, apresentam uma estruturação base em comum que preconiza informações de identificação do paciente e do profissional; dados de avaliação envolvendo habilidades e déficits do cliente (deixando claros instrumentos utilizados e demandas constatadas); plano terapêutico com metas almejadas e prazos estimados; dados sobre os atendimentos envolvendo informações sobre desempenho ocupacional, recursos empregados e observações pertinentes; resultados obtidos (incluindo dados de reavaliações) e relatório de alta com síntese do processo terapêutico sinalizando progressos obtidos, limitações e orientações recomendadas.

Entende-se que esta estruturação base que envolve informações sobre demandas, objetivos, plano terapêutico, intervenções, reavaliação e resultados, visa garantir a visibilidade do escopo da profissão e da eficácia da sua atuação. Sendo assim, a ausência ou presença de informações incompletas implicam em dificuldades de compreensão do que está sendo feito, na falta de dados para mensuração dos resultados e consequentemente em limitações na produção de pesquisas que gerem evidências científicas sobre as melhores práticas e sobre a eficácia da intervenção. Tais aspectos constituem-se como barreiras para o reconhecimento e valorização da profissão.

As anotações em prontuário exigem o domínio da linguagem técnica, a qual os profissionais nem sempre se apresentam apropriados. Nesta vertente, algumas propostas foram desenvolvidas com o intuito, entre outros, de auxiliar terapeutas ocupacionais a utilizar uma linguagem comum à profissão.

Nos EUA, a AOTA divulgou em 1994 o documento denominado "Terminologia Uniforme da Terapia Ocupacional", o qual após investimentos originou o documento "Estrutura da Prática da Terapia Ocupacional: Domínio e Processo", publicado pela primeira vez em 2002. Tais documentos fundamentam-se no modelo da ocupação e almejam o delineamento de uma linguagem comum para a práxis da Terapia Ocupacional. Sendo assim, estes documentos se constituem como referências no processo de comunicação profissional e visam favorecer a explicação da aplicabilidade de conhecimentos específicos da Terapia Ocupacional ${ }^{18}$.

Um estudo com a proposta de avaliar o nível de concordância entre os termos básicos empregados pelos terapeutas ocupacionais e os descritos na "Estrutura da Prática da Terapia Ocupacional: Domínio e Processo" (versão 2002) foi realizado com 94 terapeutas ocupacionais americanos no ano de 2005, apresentando níveis de discordância maiores que os de concordância entre os termos. Deste modo, mediante os dados empíricos, os autores referem que a terminologia e o sistema de categorização apresentado não se mostravam funcionais para os contextos da prática, da educação e da pesquisa $^{19}$. No entanto, cabe lembrar que uma nova revisão do documento foi divulgada em 2008 , não sendo encontrado em nossas buscas pesquisas voltadas a verificação do nível de concordância a partir das mudanças realizadas.

Outra ação a ser destacada refere-se ao desenvolvimento de um projeto da Rede Europeia de Terapia Ocupacional no Ensino Superior que objetivou o delineamento de uma terminologia profissional padrão. Para a efetivação desta proposta foi formado um grupo com profissionais da Áustria, Bélgica, Portugal, Espanha, Suíça e Reino Unido (representando seis línguas distintas) que trabalhou na elaboração de uma estrutura conceitual construída a partir de termos chaves utilizada pelos terapeutas ocupacionais para descreverem sua prática, originando a publicação do livro "The Core Concepts of Occupational Therapy: a Dynamic Framework for Practice" em 201020.

Pode-se afirmar que iniciativas nesta vertente ainda são pontuais e que o desenvolvimento da linguagem técnica profissional se configura como um aspecto a ser aprimorado. Para Magalhães ${ }^{21}$, a falta de clareza de conceitos dificulta o processo de internacionalização profissional e impede a valorização do trabalho desenvolvido.

Para além da apropriação profissional sobre linguagem técnica e normativas sobre os registros, se faz necessário o conhecimento sobre a importância da produção da documentação. Segundo estudo desenvolvido por Silva et al. ${ }^{22}$ na percepção dos profissionais de saúde, as principais causas das inconformidades dos registros em prontuário estão atreladas a falta de atenção e de interesse dos trabalhadores em registrar o ciclo de cuidados, a sobrecarga e déficit de recursos humanos nos serviços, ao desconhecimento dos aspectos legais da documentação e ausência de qualificação e de conhecimento sobre registros em prontuário. Tais apontamentos evidenciam lacunas no processo de educação inicial e continuada, sendo necessários investimentos nos processos de ensino aprendizagem dos cursos de graduação e de capacitações profissionais sobre domínio documental. 
A estruturação do ensino sobre documentação, seja no âmbito da graduação ou da educação continuada, deve envolver conteúdos direcionados aos aspectos técnico legais, ao aporte para desenvolvimento de indicadores de produtividade e qualidade assistencial e à oferta de direcionamentos para a efetivação de anotações consistentes que auxiliem no fomento de evidências sobre as práticas da Terapia Ocupacional. Neste planejamento, o referencial teórico utilizado para embasamento das práticas pode se tornar um recurso favorecedor ao raciocínio profissional e domínio da linguagem técnica, fatores que facilitam a sistematização das informações.

\section{CONSIDERAÇÕES FINAIS}

A prática documental no âmbito da Terapia Ocupacional apresenta-se como um problema contemporâneo que demanda investimentos do ensino de graduação, de educação continuada e da prática profissional.

As inconformidades dos registros vinculam-se ao desconhecimento das diretrizes da área, a dificuldades com a linguagem técnica e ao processo de educação inicial e continuada, sendo necessários investimentos no processo de formação dos terapeutas ocupacionais voltados a aquisição de maior domínio sobre a documentação.

Acredita-se que o processo educacional deva ofertar subsídios para ampliar a apropriação das diretrizes da profissão sobre esta temática e fundamentar-se na adoção de modelos teóricos da ocupação, os quais tendem a auxiliar a estruturação do raciocínio profissional e facilitar a descrição da prática, visto estímulos à apropriação conceituais que favorecem a linguagem técnica.

Cabe ressaltar que inconsistências nos conteúdos dos registros dificultam o desenvolvimento de pesquisas com produção de evidências robustas sobre a prática da Terapia Ocupacional, limitando o crescimento e a valorização da profissão. Sendo assim, recomenda-se a ampliação e aprofundamento de pesquisas sobre o tema, para o fomento de discussões que possibilitem a obtenção de estratégias educacionais eficazes sobre a prática do registro em prontuário e sistematizações de modelos de registros melhor gerenciáveis.

Contribuição dos autores: Tatiana Barbieri Bombarda foi responsável pelo levantamento bibliográfico, sistematização dos dados, redação do texto e revisão. Regina Helena Torkomian Joaquim foi responsável pela redação do texto e revisão.

\section{REFERÊNCIAS}

1. Santos BRP, Damian IPM. Análise da competência em informação mediante a transição do prontuário físico para o eletrônico. Rev Cub Inf Cienc Salud. 2017;28(4). doi:http:// dx.doi.org/10.36512/rcics.v28i4.1177.

2. Conselho Federal de Fisioterapia e Terapia Ocupacional. Resolução $\mathrm{n}^{\circ} 415$, de 19 de maio de 2012, que dispõe sobre a obrigatoriedade do registro em prontuário pelo terapeuta ocupacional, da guarda e do seu descarte e dá outras providências. Diário Oficial da União, Brasília, DF, n.99, Seção1, 23 maio 2012. Disponível em: https://www.coffito.gov.br/nsite/?p=3178.

3. American Occupational Therapy Association. Guidelines for documentation of occupational therapy. Am J Occup Ther. 2008;62(6):684-690. doi: https://doi.org/10.5014/ ajot.2013.67S32.

4. Matthews MM, Jabri JL. Documentação dos serviços de terapia ocupacional. In: Pedretti LM, Early MB. Terapia ocupacional: capacidades práticas para disfunções físicas. São Paulo: Roca; 2004. p.98-107.

5. College of Occupational Therapists of British Columbia. Practice Standards for Managing Client Information, 2014. Overview. Victoria, BC: COTBC; 2014 [cited 13 jul. 2018].
Available from: https://cotbc.org/wpcontent/uploads/COTBC_ ManagingClientInfo_Standards2014_ALL.pdf.

6. Possari JF. Prontuário do paciente e os registros de enfermagem. 2a ed. São Paulo: látria; 2007.

7. Marin HF, Massad E, Azevedo Neto RS. Prontuário eletrônico do paciente: definições e conceitos. In: Massad E, Marin HF, Azevedo Neto RS, et al. O prontuário eletrônico do paciente na assistência, informação e conhecimento médico. São Paulo: H. de F. Marin; 2003. p.1-20.

8. College of Occupational Therapists of Ontario. Practice Guideline: client records. Ontario; 1999 [cited 28 mar. 2019]. Available from: https:/www.coto.org/docs/default-source/ default-document-library/record-keeping-standard-jan1. pdf? sfvrsn $=10$.

9. Pierre BL, Sonn U. Occupational therapy as documented in patient's records: Part II: What is proper documentation? Contradictions and aspects of concern form the perspective of OTs. Scand J Occup Ther. 1999;6:3-10. doi: https://doi. org/10.3109/11038129609106687.

10. Pierri BL. Occupational Therapy as Documented in Patients Records Part III. Valued but not Documented. Underground Practice in the Context of Professional Written 
Communication. Scand J Occup Ther. 2001;8(4):174-83. doi: https://doi.org/10.1080/110381201317166531.

11. Hedberg-Kristensson E, Iwarsson S. Documentation quality in occupational therapy patients records: focusing on the technical aid prescription process. Scand J Occup Ther. 2003;10:72-80. doi: https://doi.org/10.1080/11038120310009434.

12. Rischmuller R, Franzsen D. Assessment of record keeping at schools for learners with special educational needs in the Western Cape. S Afr J Occup Ther. 2012;42(2):13-20. Available from: http://www.scielo.org.za/scielo.php?script=sci arttext\&pid=S2310-38332012000200005\&lng=en.

13. Bombarda TB, Palhares MS. O registro de práticas interventivas da terapia ocupacional na educação inclusiva. Cad Bras Ter Ocup. 2015;23(2):285-94. doi: https://doi.org/10.4322/ 0104-4931.ctoAO0496.

14. Pelissari DC, Palhares MS. O registro da intervenção no prontuário pelo terapeuta ocupacional em um ambulatório infanto-juvenil. Cad Bras Ter Ocup. 2015;23(4):711-22. doi: https://doi.org/10.4322/0104-4931.ctoAO0497.

15. Davis $\mathrm{J}$, et al. Communicating evidence in clinical documentation. Aust Occup Ther J. 2008;55:249-55. doi: https://doi.org/10.1111/j.1440-1630.2007.00710.x.

16. Buchanan H, Jelsma J, Siegried N. Practice-based evidence: evaluating the quality of occupational therapy patient records as evidence for practice. S Afr J Occup Ther. 2016;46(1):65-73. doi: http://dx.doi.org/10.17159/2310-3833/2016/v46n1a13.

17. Gillete NP. A data base for occupacional therapy: documentation through research. Am J Occup Ther. 1982;36(8):499-501. https://doi.org/10.5014/ajot.36.8.499.

18. Drumond AF. Fundamentos da terapia ocupacional. In: Cavalcanti A, Galvão C. Terapia ocupacional: fundamentação e prática. Rio de Janeiro: Guanabara Koogan; 2007. p.10-17.

19. Butts DS, Nelson DL. Agreement between Occupational Therapy Practice Framework Classifications and Occupational Therapists' Classifications. Am J Occup Ther. 2007;61(5):512-8. doi: https://doi.org/10.5014/ajot.61.5.512.

20. Creek J. The core concepts of occupational therapy: a dynamic framework for practice. London: Jessica Kingsley Publishers; 2010.

21. Magalhães L. Ocupação e atividade: tendências e tensões conceituais na literatura anglófona da terapia ocupacional e da ciência ocupacional. Cad Bras Ter Ocup. 2013;21(2):255-63. doi: https://doi.org/10.4322/cto.2013.027.

22. Silva LCS, et al. Inconformidades acerca dos registros em prontuários: percepção dos trabalhadores de saúde da região central do Brasil. Atas CIAIQ. 2017;2:1570-7 [acesso em 04 de março 2019]. Disponível em: https://proceedings.ciaiq.org/ index.php/ciaiq2017/article/view/1509. 